\title{
Somatic Membrane Potential and Kv1 Channels Control Spike Repolarization in Cortical Axon Collaterals and Presynaptic Boutons
}

\author{
Amanda J. Foust, ${ }^{1}$ Yuguo Yu, ${ }^{1,3}$ Marko Popovic, ${ }^{2}$ Dejan Zecevic, ${ }^{2}$ and David A. McCormick ${ }^{1}$ \\ Departments of ${ }^{1}$ Neurobiology, Kavli Institute for Neuroscience, and ${ }^{2}$ Cellular and Molecular Physiology, Yale University School of Medicine, New Haven, \\ Connecticut 06510, and ${ }^{3}$ Center for Computational Systems Biology, Fudan University, Shanghai, People's Republic of China 200433
}

The shape of action potentials invading presynaptic terminals, which can vary significantly from spike waveforms recorded at the soma, may critically influence the probability of synaptic neurotransmitter release. Revealing the conductances that determine spike shape in presynaptic boutons is important for understanding how changes in the electrochemical context in which a spike is generated, such as subthreshold depolarization spreading from the soma, can modulate synaptic strength. Utilizing recent improvements in the signal-to-noise ratio of voltagesensitive dye imaging in mouse brain slices, we demonstrate that intracortical axon collaterals and en passant presynaptic terminals of layer 5 pyramidal cells exhibit a high density of $\mathrm{Kvl}$ subunit-containing ion channels, which generate a slowly inactivating $\mathrm{K}^{+}$current critically important for spike repolarization in these compartments. Blockade of the current by low doses of 4 -aminopyridine or $\alpha$-dendrotoxin dramatically slows the falling phase of action potentials in axon collaterals and presynaptic boutons. Furthermore, subthreshold depolarization of the soma broadened action potentials in collaterals bearing presynaptic boutons, an effect abolished by blocking Kv1 channels with $\alpha$-dendrotoxin. These results indicate that action potential-induced synaptic transmission may operate through a mix of analog-digital transmission owing to the properties of Kvl channels in axon collaterals and presynaptic boutons.

\section{Introduction}

The computation performed by a single cortical neuron depends upon the distribution and properties of ionic channels in its axon collaterals, since these determine the shape of action potentials in local presynaptic terminals, the rate and amplitude of calcium influx, and subsequently the probability of neurotransmitter release. Indeed, the kinetics of single action potentials and transmitter release probability are sensitive to recent history and network state, suggesting that spikes participate in interplexed modes of analog and digital communication (Alle and Geiger, 2006; Kole et al., 2007; Shu et al., 2007; Christie et al., 2011). For example, subthreshold somatic depolarization increases the average amplitude of the EPSP evoked by isolated action potentials (Shu et al., 2006). Recordings from the cut ends of primary axons show that this facilitation of postsynaptic responses may be accompanied by an increase in axonal spike width mediated through inactivation of Kv1 channels. Importantly, low doses of either 4-aminopyridine or Kv1.1, 1.2, and 1.6 channel blocker

Received June 1, 2011; revised Sept. 6, 2011; accepted Sept. 7, 2011.

Author contributions: A.J.F., Y.Y., M.P., D.Z., and D.A.M. designed research; A.J.F., Y.Y., and M.P. performed research; A.J.F. and Y.Y. analyzed data; A.J.F. and D.A.M. wrote the paper.

This work was supported by the National Institutes of Health (D.A.M., D.Z.), the National Science Foundation (NSF) (D.Z.), an NSF graduate research fellowship (A.J.F.), a Ruth L. Kirschstein National Research Service Award (A.J.F), and the Kavli Institute for Neuroscience at Yale. We thank Matthew McGinley (Yale University, New Haven, (T) for valuable help with statistics.

Correspondence should be addressed to David A. McCormick, Kavli Institute for Neuroscience, Yale University School of Medicine, 333 Cedar Street, New Haven, CT 06510. E-mail: David.mccormick@yale.edu.

DOI:10.1523/JNEUROSCI.2752-11.2011

Copyright $\odot 2011$ the authors $\quad 0270-6474 / 11 / 3115490-09 \$ 15.00 / 0$ $\alpha$-dendrotoxin ( $\alpha$-DTX) strongly influence cortical synaptic transmission and dramatically increase the duration of action potentials in the primary axon of layer 5 pyramidal cells (Kole et al., 2007; Shu et al., 2007). However, it is unknown whether en passant presynaptic terminals located on axon collaterals also contain Kvl channels and propagate broadened spikes during subthreshold somatic depolarization.

What currents determine the shape of action potentials as they invade cortical presynaptic terminals? Due to the small size of axon collaterals and presynaptic boutons, previous electrophysiological investigations in the neocortex have been limited to patch-clamp recordings of large presynaptic structures, indirect analysis of EPSCs recorded at the soma, and calcium imaging. Patch recordings of large presynaptic structures have demonstrated the presence of both transient and persistent sodium currents (Ahern et al., 2000; Engel and Jonas, 2005; Leão et al., 2005; Huang and Trussell, 2008). Moreover, axonal voltage-gated potassium channels exhibit great diversity (Dodson and Forsythe, 2004). For example, low-threshold-activated Kv1 channels, which protect presynaptic terminals from aberrant spike generation, occupy axon terminals in structures as diverse as hippocampus, brainstem, and thalamus (Wang et al., 1994; Cooper et al., 1998; Southan and Robertson, 2000; Lambe and Aghajanian, 2001; Monaghan et al., 2001; Dodson et al., 2003). Kv3-subunitcontaining $\mathrm{K}^{+}$conductances are also prominent in axons, particularly those of fast-spiking interneurons (Weiser et al., 1995) and other cell types (Southan and Robertson, 2000; Dodson et al., 2003; Dallas et al., 2005), and serve to limit action potential duration. The small size of cortical synaptic terminals has precluded 
direct characterization of potassium subunits responsible for spike repolarization in these structures, and how these currents are modified by subthreshold voltage perturbations spreading passively in the main axon.

Here, through the use of a recent significant advance in voltagesensitive dye (VSD) imaging (Canepari et al., 2010; Holthoff et al., 2010), we show that steady-state somatic membrane potential modulates the shape of action potentials invading local presynaptic terminals through the inactivation of Kv1, but not Kv3, subunit-containing $\mathrm{K}^{+}$channels.

\section{Materials and Methods}

Slice preparation and maintenance. We prepared slices of somatosensory cortex from transgenic mice (CRYM-EGFP) in accordance with the guidelines of the Yale University Institutional Animal Care and Use Committee. Male or female mice (2-5 weeks of age) were deeply anesthetized with sodium pentobarbital $(50 \mathrm{mg} / \mathrm{kg})$ and killed through decapitation. The brain was rapidly removed and placed in ice-cold $\left(<5^{\circ} \mathrm{C}\right)$ cutting solution containing the following (in $\mathrm{mm}$ ): 110 choline chloride, $2.5 \mathrm{KCl}, 7.0 \mathrm{MgSO}_{4}, 0.5 \mathrm{CaCl}_{2}, 25 \mathrm{NaHCO}_{3}, 1.25 \mathrm{NaH}_{2} \mathrm{PO}_{4}, 20$ glucose, and aerated with $95 \% \mathrm{O}_{2}, 5 \% \mathrm{CO}_{2}$ to a final $\mathrm{pH}$ of 7.4. We dissected the cerebral cortex, blocking it such that the slices of somatosensory cortex would preserve thalamocortical projections (Agmon and Connors, 1991). The cortical block was glued to an ice-cold stage on a Leica microslicer, and 300-400 $\mu \mathrm{m}$ slices were cut in ice-cold cutting solution. The sections were placed in an incubator at $35^{\circ} \mathrm{C}$ for $0.5 \mathrm{~h}$ in the cutting solution, and were then transferred to a chamber containing ACSF (in mм: $126 \mathrm{NaCl}, 3.0 \mathrm{KCl}, 1.0 \mathrm{MgSO}_{4}, 2.0 \mathrm{CaCl}_{2}, 26 \mathrm{NaHCO}_{3}, 1.25$ $\mathrm{NaH}_{2} \mathrm{PO}_{4}, 10$ glucose, 3 myo-inositol, $2 \mathrm{Na}$-pyruvate, and $0.4 \mathrm{~L}$-ascorbic acid) at room temperature for at least $0.5 \mathrm{~h}$ before dye loading and recording in a submersion chamber.

Cell selection. The voltage-sensitive dye imaging system is constructed around an upright microscope (Olympus BX51WI) equipped to switch easily between three different imaging systems: (1) confocal epifluorescence for cell selection and morphological reconstruction (Yokogawa CSU-XAH system, Solamere Tech); (2) IR-DIC video microscopy for establishing whole-cell recordings; and (3) wide-field epifluorescence for voltage-sensitive dye imaging. Initially, CRYM-EGFP slices were examined with the confocal imaging system for morphological completeness of their dendritic and especially axonal processes, using a three-line argon laser at $50 \mathrm{~mW}, 488 \mathrm{~nm}$ (National Laser Company). This process of cell selection illuminated individual neurons typically for $<1 \mathrm{~min}$ and was determined to be nontoxic (Foust et al., 2010).

The signal-to-noise $(\mathrm{S} / \mathrm{N})$ ratio in voltage-sensitive dye recording depends, among other factors, on the amount of dye filling the cell, the morphology of the cell, and the proximity of the cell to the surface of the slice. Because we image in wide-field mode, all structures to be simultaneously monitored must lie close to the plane of focus. The closer the imaged structures lie to the surface of the slice, the less the image is degraded by light scattering in the tissue. The selection of neurons with processes that are intact and anatomically appropriate for imaging has been one of the major limiting steps in experiments using VSD recording because thin neuronal processes are not visible under DIC videomicroscopy. By using transgenic mouse lines expressing EGFP, we were able to select neurons with optimal morphology before dye loading, greatly increasing the efficiency of these experiments. The EGFP fluorescence does not interfere with voltage-sensitive dye signals due to the non-overlapping emission spectra of these two fluorophores.

Voltage-sensitive dye loading and recording. We loaded selected neurons with voltage-sensitive dye with a glass patch electrode by filling the tip with dye-free internal solution (in mM: $130 \mathrm{~K}$-gluconate, $7 \mathrm{KCl}, 4 \mathrm{ATP}-\mathrm{Mg}, 0.3$ GTP-Na, 10 phosphocreatine-Na, 10 HEPES, and $0.4 \%$ biocytin; adjusted to $\mathrm{pH} 7.4$ and $284 \mathrm{~mol} / \mathrm{kg}$ ), then back-filling with the dye-containing internal solution (JPW3028, 400-800 $\mu \mathrm{M}$ ), and performed whole-cell somatic recordings from the selected neuron under DIC optics. The dye JPW3028 is a doubly positively charged analog of the aminostyryl-pyridinium series of lipophilic voltage-sensitive dyes available from Invitrogen as D-6923. This electrochromic dye does not significantly increase the membrane capaci- tance of the labeled neuron, as evident from several control measurements showing that the waveform of the electrically recorded action potentials remains unaltered after intracellular application of the dye (e. g., Canepari et al., 2007). Dye-free solution in the tip is necessary because this highly lipophilic dye would otherwise spill onto the slice before sealing and patching the cell of interest. Any dye bound to membrane outside the cell of interest increases the background fluorescence and thus degrades recording sensitivity. The selected cell was patched and stained at room temperature for 20-50 min by allowing passive diffusion of the dye into the cell. After staining, we carefully detached the patch electrode from the neuron, forming an outsideout patch, after which the slice was incubated at room temperature for $1-4 \mathrm{~h}$, allowing the dye to diffuse from the soma into the distal axon and axon collaterals. The dye diffuses slowly compared with other dyes of the same size/weight due to its high affinity for lipid membranes. Before recording, the perfusion solution temperature was typically increased to physiological level $\left(35-36^{\circ} \mathrm{C}\right)$, and we repatched the stained cell with a voltage-sensitive dye-free electrode for simultaneous optical and electrophysiology recording.

Cells filled with the voltage-sensitive dye were illuminated with a $450 \mathrm{~mW}$ frequency-doubled diode-pumped Nd:YVO4 low-noise laser emitting at $532 \mathrm{~nm}$ (MLL532, Changchun New Industries Optoelectronics Tech). The laser beam was directed to a light guide coupled to the microscope via a single-port epifluorescence condenser (TILL Photonics) designed to overfill the back aperture of the objective. In this way, near-uniform illumination of the object plane was attained. Illumination of the soma was limited by the microscope field-stop to minimize unnecessary damage to nonimaged regions of the recorded neuron. Fluorescence from the stained cell was collected through the objective $(60 \times$ water immersion, Nikon), long-pass filtered (RG610 HP, Omega Optical), and projected onto a high-speed CCD camera recording at a $10 \mathrm{kHz}(80 \times 12$ pixels $)$ or $20 \mathrm{kHz}(26 \times 4$ pixels $)$ frame rate (NeuroCCD-SMQ, RedShirtImaging) mounted at the front camera port of the trinocular tube. At the magnification that we used, each pixel of the camera corresponded to $1.2 \times 1.2 \mu \mathrm{m}(10 \mathrm{kHz}$ frame rate $)$ or $3.6 \times 3.6$ $\mu \mathrm{m}(10-20 \mathrm{kHz}$ frame rate) surface area in the object plane.

Changes in voltage-sensitive dye fluorescence were recorded during action potentials evoked with single $5 \mathrm{~ms}$ current pulses injected into the soma. The resting membrane potential was adjusted on alternate trials between a hyperpolarized membrane potential $(-72$ to $-70 \mathrm{mV})$ and a depolarized membrane potential just below firing threshold $(-52$ to $-48 \mathrm{mV})$ for $>10 \mathrm{~s}$ before the imaged spike. Values reported for membrane potential are not corrected for liquid junctional potential. After 6-20 trials were recorded in control ACSF, a voltage-gated potassium channel antagonist, tetraethyl ammonium (TEA, $1 \mathrm{~mm}$ ), 4-aminopyridine (4-AP, $40 \mu \mathrm{M}$ ), or $\alpha$-dendrotoxin $(\alpha$-DTX, $100 \mathrm{nM})$, was added to the bath solution. Slices were perfused with drug-containing solution for $10 \mathrm{~min}$ before measurements. Each imaging trial lasted $\sim 10-40 \mathrm{~ms}$, and the stained neuron was illuminated only during this period. We could obtain between 15 and 25 trials before changes in the electrophysiological properties of the recorded neuron (action potential amplitude and duration, resting membrane potential, and resting input resistance) were evident, in which case the experiment was terminated.

Data analysis. The $\mathrm{S} / \mathrm{N}$ obtained during voltage-sensitive dye imaging varied from cell to cell depending on the concentration of dye and proximity of the axon to the surface of the slice. Thus, to achieve comparable $\mathrm{S} / \mathrm{N}(>10: 1)$ ratio across experiments, we averaged fluorescence changes from groups of neighboring pixels. Although we could often obtain this $\mathrm{S} / \mathrm{N}$ ratio in single-trial recordings, we performed temporal averaging of a small number (4-32) of trials to maximize the accuracy of measurement of spike width and rise time. To avoid smearing in the trial-to-trial average due to temporal jitter, for each trial we aligned the signal in each pixel to the peak of the electrically recorded action potential (AP) from the soma. A low-pass binomial filter (1-2 passes) was applied to reduce high-frequency noise. Slow changes in fluorescence intensity due to bleaching of the dye were removed by subtracting a biexponential function fit to portions of the fluorescence signal containing no spikes. Because the $\Delta F / F$ signal from different parts of the neuron varies owing to factors other than voltage (e.g., partitioning of dye in inner and outer membranes), changes in voltage indicated by changes in fluorescence could not be calibrated on an absolute scale. However, the conclusions of our study do not depend on precise calibration of membrane potential signals on an absolute voltage scale. 
To characterize action potential kinetics in the axon initial segment (AIS), main axon, axon collaterals, and presynaptic boutons, we measured spike width (full-width at half-maximum, FWHM) and rise time ( $10 \%$ to $90 \%)$ and fall time ( $90 \%$ to $20 \%$ ) of action potential-related fluorescence signals from each of these compartments. Data were acquired at a frame rate of 10 or $20 \mathrm{kHz}$ and the waveform of the AP signal was reconstructed using cubic spline interpolation. The interpolated signal was resampled at $100 \mathrm{kHz}$ before measurement of spike width, rise time, and fall time. To compare the kinetics of action potentials in different regions of the neuron, we scaled all spikes to the same baseline and peak height. Although spike fluorescence amplitude cannot be compared across pixels due to variable background fluorescence, the relative amplitude of spike-evoked fluorescence changes was calculated across variable conditions (i.e., soma hyperpolarized vs soma depolarized) in the same group of pixels. At the end of the experiment, we obtained a detailed three-dimensional morphology of the imaged axon by taking a $z$-stack of images through the axonal arbor with the confocal microscope.

Pairs of datasets were compared with paired Student's $t$ tests. ANOVA was used to compare more than two groups, and repeated-measures ANOVA was used when appropriate. Groupwise $p$ values and $F$ values are reported when ANOVA was used; pairwise comparisons were made using Tukey's post hoc. All analyses and statistics were performed in NeuroPlex (RedShirtImaging), Origin, and Pylab.

Methods for computational model of action potential propagation in cortical neurons. To examine the effect of D-current on spike broadening in axon collaterals, we simulated the initiation and propagation of action potentials in two model axons: one with D-current in the AIS, nodes and collateral, and another with D-current in the AIS only. The models each consist of a cylindrical axon (500 $\mu \mathrm{m}$ length) with a $20 \mu \mathrm{m}$ AIS ( $1.2 \mu \mathrm{m}$ diameter) connected to a myelinated axon (1.4 $\mu \mathrm{m}$ diameter) with nodes (each $2 \mu \mathrm{m}$ length, 1.2 $\mu \mathrm{m}$ diameter) at 100 and $300 \mu \mathrm{m}$. A collateral is attached to the first node of Ranvier (500 $\mu \mathrm{m}$ length, $0.5 \mu \mathrm{m}$ diameter, 100 compartments) issued 80 $\mu \mathrm{m}$ from the AIS (Fig. 4A). Our models were implemented in NEURON (Hines and Carnevale, 1997) at a temperature of $37^{\circ} \mathrm{C}$ with internal resistance, $R_{2}$, of $150 \Omega \cdot \mathrm{cm}$, specific membrane capacitance of $0.04 \mu \mathrm{F} / \mathrm{cm}^{2}$ in myelinated segments and $0.8 \mu \mathrm{F} / \mathrm{cm}^{2}$ elsewhere, and $g_{\text {leak }}$ of $0.33 \mathrm{pS} / \mu \mathrm{m}^{2}$. The density of $g_{\mathrm{Na}}$ is $4000 \mathrm{pS} / \mu \mathrm{m}^{2}$ in the AIS, $2800 \mathrm{pS} / \mu \mathrm{m}^{2}$ in the nodes, $1333 \mathrm{pS} / \mu \mathrm{m}^{2}$ in the collaterals, and $10 \mathrm{pS} / \mu \mathrm{m}^{2}$ in the myelinated segments based on recent experimental findings (Colbert and Pan, 2002; Kole et al., 2008; Hu et al., 2009). The distribution of delayed rectifier potassium conductances with Hodgkin-Huxley-style kinetics $\left(g_{\mathrm{Kv}}\right)$ was adjusted to a low level in the D-current model to match experimental recordings (Kole et al., 2007; Shu et al., 2007) and theoretical predictions (Shu et al., 2007; Hasenstaub et al., 2010) in cortical pyramidal neurons: $g_{\mathrm{Kv}}$ is $6 \mathrm{pS} / \mu \mathrm{m}^{2}$ in the AIS, $3 \mathrm{pS} / \mu \mathrm{m}^{2}$ in nodes of Ranvier, and $10 \mathrm{pS} / \mu \mathrm{m}^{2}$ in the collateral. We explored the impact of a wide range of parameters on the ability of a $15-20 \mathrm{mV}$ somatic membrane potential depolarization to affect action potential duration in axon collaterals, including the ratio of $I_{\mathrm{Kv}}$ to $I_{\mathrm{KD}}$, and the density and distribution of $I_{\mathrm{KD}}$ in the AIS, main axon, and collateral. The most critical parameter for replicating our experimental finding of spike broadening in axon collateral with somatic depolarization was the density and distribution of the conductance underlying the rapidly activating, slowly inactivating $\mathrm{K}^{+}$ current $I_{\mathrm{KD}}$ or $I_{\mathrm{D}} \cdot g_{\mathrm{Kd}}$ was distributed in the AIS, primary axon, and collaterals such that the model exhibited action potential broadening with longlasting, subthreshold depolarization, as observed previously in the main axon (Kole et al., 2007; Shu et al., 2007) and axon collateral (present study): $g_{\mathrm{Kd}}$ is $150 \mathrm{pS} / \mu \mathrm{m}^{2}$ in the AIS, $72 \mathrm{pS} / \mu \mathrm{m}^{2}$ in the nodes of Ranvier, and 60 $\mathrm{pS} / \mu \mathrm{m}^{2}$ in the axon collateral. In our second model in which there is no $\mathrm{D}$-current in the axon collateral, the density of spike-repolarizing $\mathrm{K}^{+}$currents was as follows: $g_{\mathrm{Kv}}=6 \mathrm{pS} / \mu \mathrm{m}^{2}$ and $g_{\mathrm{Kd}}=150$ (or $210 \mathrm{pS} / \mu \mathrm{m}^{2}$ ) in the AIS, $g_{\mathrm{Kv}}=100 \mathrm{pS} / \mu \mathrm{m}^{2}$ and $g_{\mathrm{Kd}}=0 \mathrm{pS} / \mu \mathrm{m}^{2}$ in the nodes of Ranvier, and $g_{\mathrm{Kv}}=60 \mathrm{pS} / \mu \mathrm{m}^{2}$ and $g_{\mathrm{Kd}}=0 \mathrm{pS} / \mu \mathrm{m}^{2}$ in the axon collateral. Note that the lack of a broadening effect of AIS depolarization on the action potential in the axon collateral was not dependent on the increased level of $g_{\mathrm{Kv}}$ in the collaterals. The same effects were observed whether $g_{\mathrm{Kv}}$ was 60 or $10 \mathrm{pS} / \mu \mathrm{m}^{2}$. The following equations describe the voltage and time dependence of the
$\mathrm{Na}^{+}$and $\mathrm{K}^{+}$conductances (McCormick, 1992; Mainen et al., 1995; Yu et al., 2008):

$$
\begin{aligned}
& I_{\mathrm{Na}}=g_{\mathrm{Na}} m^{3} h\left(V-E_{\mathrm{Na}}\right) \\
& I_{\mathrm{K}}=g_{\mathrm{K}} n\left(V-E_{\mathrm{K}}\right) \\
& I_{1}=g_{l}\left(V-E_{l}\right) \\
& \frac{d m}{d t}=-\frac{m-m_{\infty}}{\tau_{m}}, \tau_{m}=\frac{1}{\alpha_{m}+\beta_{m}}, m_{\infty}=\frac{\alpha_{m}}{\alpha_{m}+\beta_{m}} \\
& \frac{d h}{d t}=-\frac{h-h_{\infty}}{\tau_{h}}, \tau_{h}=\frac{1}{\alpha_{h}+\beta_{h}}, h_{\infty}=\frac{1}{1+e^{(V+60) / 6.2}} \\
& \frac{d n}{d t}=-\frac{n-n_{\infty}}{\tau_{n}}, \tau_{m}=\frac{1}{\alpha_{n}+\beta_{n}}, m_{\infty}=\frac{\alpha_{n}}{\alpha_{n}+\beta_{n}} \\
& \alpha_{m}(V)=\phi \cdot \frac{0.182 \cdot(V+30)}{1-e^{-(V+30) / 8}} \\
& \beta_{m}(V)=-\phi \cdot \frac{0.124 \cdot(V+30)}{1-e^{(V+30) / 8}} \\
& \alpha_{h}(V)=\phi \cdot \frac{0.028 \cdot(V+45)}{1-e^{-(V+45) / 6}} \\
& \beta_{h}(V)=-\phi \cdot \frac{0.0091 \cdot(V+70)}{1-e^{(V+70) / 6}} \\
& \alpha_{n}(V)=\phi \cdot \frac{0.01 \cdot(V-30)}{1-e^{-(V-30) / 9}} \\
& \beta_{n}(V)=-\phi \cdot \frac{0.002 \cdot(V-30)}{1-e^{(V-30) / 9}} \\
& \phi=Q_{10}^{(T-23) / 10},
\end{aligned}
$$

where the $Q_{10}$ effect, described by $\Phi$, regulates temperature dependence, with $Q_{10}=2.3$.

The following equations describe the voltage and time dependence of D-current (Shu et al., 2007):

$$
\begin{gathered}
I_{\mathrm{Kd}}=g_{\mathrm{Kd}} m_{\mathrm{Kd}} h_{\mathrm{Kd}}\left(V-E_{\mathrm{Kd}}\right) \\
\frac{d m_{\mathrm{Kd}}}{d t}=-\frac{m_{\mathrm{Kd}}-m_{\mathrm{Kd}}^{\infty}}{\tau_{m}} \\
\frac{d h_{\mathrm{Kd}}}{d t}=-\frac{h_{\mathrm{Kd}}-h_{\mathrm{Kd}}^{\infty}}{\tau_{h}}
\end{gathered}
$$

where:

$$
\begin{gathered}
m_{\mathrm{Kd}}^{\infty}=1-\frac{1}{1+\exp \left(V-V_{1 / 2}^{m}\right) / q_{m}} \\
h_{\mathrm{Kd}}^{\infty}=1-\frac{1}{1+\exp \left(V-V_{1 / 2}^{h}\right) / q_{h}} \\
\tau_{m}=1(\mathrm{~ms}) \\
\tau_{h}=1500(\mathrm{~ms}) \\
V_{1 / 2}^{m}=-43(\mathrm{mV}) \\
V_{1 / 2}^{h}=-67(\mathrm{mV}) \\
q_{m}=8 \\
q_{h}=7.3 .
\end{gathered}
$$


A

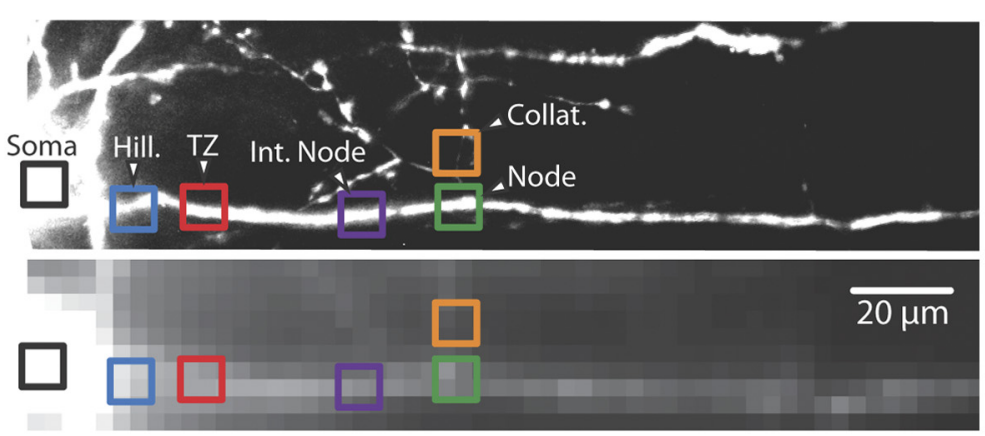

B

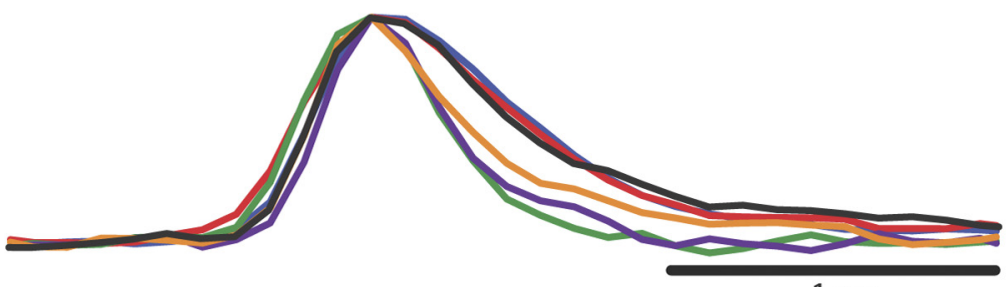

$1 \mathrm{~ms}$
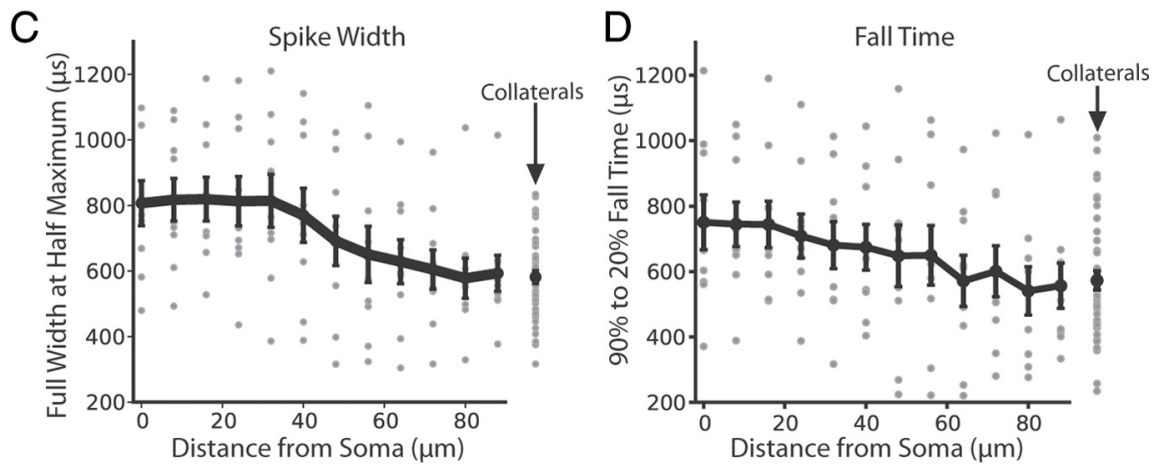

Figure 1. Basic properties of axonal action potentials. Action potentials become thinner as they propagate through nodes, internodes, and collaterals. $A$, A voltage-sensitive dye fluorescence image of a layer 5 pyramidal neuron generated from stacks of images obtained with a spinning-disc confocal scanner (top) and CCD camera for voltage imaging (bottom). $\boldsymbol{B}$, Fluorescence transients averaged over pixels outlined by the same-colored boxes in $A$ showing the characteristic spike shape in the hillock, TZ, node, internode and collateral. Traces are scaled to the same height. C, Spike width (full-width at half-maximum amplitude) plotted as a function of distance from the soma in the main axon ( $n=9$ cells). The black line shows the mean and SE, and the gray points show data from individual cells. The rightmost data point shows mean spike width in the collaterals. $\boldsymbol{D}$, Fall time $(90 \%$ to $10 \%$ ) plotted as a function of distance from the soma in the main axon ( $n=9$ cells). The black line shows the mean and SEM, and the gray points show data from individual cells. The rightmost data point shows mean spike width in the collaterals.

The source code for our models is available from ModelDB (http://modeldb.yale.edu).

Subthreshold depolarization was simulated as a $20 \mathrm{~s}, 60$ pA DC injection at the AIS, and individual spikes were evoked at the beginning and end of the DC injection with $5 \mathrm{~ms}, 140 \mathrm{pA}$ current pulses at the AIS. As for experimental data, spike widths were measured as the full-width at halfmaximal amplitude.

\section{Results}

To examine the kinetics of axonal action potentials as they propagate, we recorded changes in voltage-sensitive dye fluorescence in the AIS, main axon, and collaterals of layer 5 pyramidal neurons in somatosensory cortical slices. Each cell was selected for long $(>200$ $\mu \mathrm{m})$, intact axonal arbors lying in a single plane using a spinning disk confocal system to examine EGFP-labeled neurons (CRYM mouse line) before staining with voltage-sensitive dye. Cells with enough of their main axons and axonal arbors intact and near the upper surface of the slice to reveal at least primary, secondary, or higher levels of axon collaterals were loaded with voltage-sensitive dye JPW3028 as previously described (Antic et al., 1999; Djurisic et al., 2004; Cane- pari et al., 2008; Foust et al., 2010). After loading and a $1-4 \mathrm{~h}$ resting period, we repatched the cell with an electrode filled with control (no dye) internal solution. Dye loading caused no significant change in cell input resistance (prestaining: $86.8 \pm 8.3$ $\mathrm{M} \Omega$ vs stained: $98.8 \pm 12.1 \mathrm{M} \Omega, n=5$, paired Student's $t$ test $p>0.1$ ). Comparisons between control cells and dye-filled cells at $35^{\circ} \mathrm{C}$ revealed no significant differences in action potential parameters including amplitude (control: $70.8 \pm 2.24 \mathrm{mV}$, $n=11$ vs stained: $71.7 \pm 1.1 \mathrm{mV}, n=13$; $p>0.3$ unpaired Student's $t$ test), width (control: $1.00 \pm 0.05 \mathrm{~ms}, n=11$ vs stained: $1.01 \pm 0.03 \mathrm{~ms}, n=13 ; p>0.3)$, and maximum $d V / d t$ (control: $308.1 \pm 21.5 \mathrm{~V} / \mathrm{s}, n=$ 11 vs stained: $301.1 \pm 7.6 \mathrm{~V} / \mathrm{s}, n=13 ; p>$ 0.3 ) as previously reported in other cell types (Djurisic et al., 2004; Canepari et al., 2007; Foust et al., 2010). We recorded action potentials electrically with the somatic patch electrode and optically by imaging changes in fluorescence $(\Delta F / F)$ throughout the main axon and axon collaterals. Recent advances in the sensitivity of the voltagesensitive dye imaging technique based on monochromatic laser light excitation enabled us to record at the high frame rates $(10-20 \mathrm{kHz})$ necessary to detect slight changes in action potential shape across locations and experimental conditions.

\section{Basic properties of axonal action potential propagation}

To characterize changes in the waveform of action potentials as they initiate and propagate throughout the axonal arbor, we measured fall time (90 to 20\%) and width (FWHM) of fluorescence transients in the axon hillock, spike trigger zone (TZ, located in the distal AIS), node, internode, and collaterals (Fig. 1A) generated in response to intrasomatic injection of shortduration ( $5 \mathrm{~ms}$ ) depolarizing current pulses. Fluorescence signals were averaged temporally (4-50 trials) and spatially (2 pixels covering $8 \mu \mathrm{m}$ ). The hillock was identified anatomically as the first 5-10 $\mu \mathrm{m}$ of axon directly attached to the soma. The region labeled TZ in Figure 1 denotes the region of distal AIS where the fluorescence signal reaches $20 \%$ of maximal amplitude first, that is, the location brought to spike threshold first. Since spikes generated in axonal nodes rise faster than adjoining internodal regions (Foust et al., 2010), the first node of Ranvier was identified functionally as the region located $60-140 \mu \mathrm{m}$ exhibiting a local minimum in spike onset latency (measured at $20 \%$ of maximum amplitude). In most cases, functionally identified nodes corresponded anatomically to the issuance of the first collateral branch. We found that action potentials decreased in width and fall time as they propagated orthodromically from the trigger zone through the main axon (Fig. 1C,D) in agreement with previous measurements (Kole et al., 2007). In addition, the fall times and widths of action potentials measured in axon collaterals (width, FWHM: mean $=581.8 \pm 19.9 \mu$ s; $90 \%$ to $20 \%$ fall time: 
$572.8 \pm 29.4 \mu \mathrm{s}, n=41)$ were similar to the distal main axon (Fig. 1C,D). These results suggest that the currents underlying spike generation, in combination with morphological and passive electrical properties, may vary significantly across the different portions of the soma-axonal topography (Kole et al., 2007; Shu et al., 2007).

\section{Steady-state subthreshold}

depolarization of soma causes distancedependent action potential broadening Broadening of action potentials observed in the main axon of layer 5 pyramidal neurons has been proposed as a mechanism through which subthreshold depolarization of a presynaptic neuron may facilitate average postsynaptic responses (Kole et al., 2007; Shu et al., 2007). However, it was unknown whether subthreshold somatic depolarization increases action potential width in axon collaterals, which contain the en passant boutons participating in local synaptic transmission. To examine effects of steadystate subthreshold depolarization of the soma on axonal action potential shape, we recorded spike-evoked fluorescence transients in axon collaterals generated in response to brief intrasomatic current injections. Each stimulus was preceded by a long $(>10 \mathrm{~s})$ hyperpolarization $\left(V_{\mathrm{ss}}=-72\right.$ to $-70 \mathrm{mV}$ ) or subthreshold depolarization ( $V_{\mathrm{ss}}=-52$ to $-48 \mathrm{mV}$ ) applied with $\mathrm{DC}$ injection through the somatic whole-cell electrode on alternate trials. The signal-tonoise ratio was improved by averaging signals from pixels covering contiguous, unbranched 10-50 $\mu \mathrm{m}$ lengths of axon across hyperpolarized and depolarized trials. We found that subthreshold somatic depolarization decreased the amplitude (Fig. $2 C$ ) and increased the rise time (Fig. $2 B, C$ ), the fall time (Fig. $2 B, C$ ), and the width (Fig. $2 B-D)$ of action potentials recorded with the patch electrode in the soma (mean $=15.4 \pm 1.0 \%$ change in width, $n=41$ cells) and imaged in axon collaterals (mean $=12.6 \pm 2.2 \%$ change in width, $n=50$ collaterals). Action potential broadening was negatively correlated with the total distance $\left(D_{\text {tot }}=D_{\text {main axon }}+D_{\text {collat }^{-}}\right.$ eral) of axon between soma and recording site center, with effects in the range of $\sim 10-40 \%$ broadening occurring at the collateral locations nearest to the soma (e.g., $\sim 100-125 \mu \mathrm{m}$ ) (Fig. $2 E$ ). This effect decreased with distance, and could be fit with a linear regression model, predicting that the broadening effect would reach zero within $350 \mu \mathrm{m}$ from the soma (Fig. $2 E$ ). No significant correlation was observed between the number of branch points between soma and recording site and the amplitude of depolarization-induced spike broadening.

Kv1 subunit-containing channel blockade causes spike broadening in axon collaterals and presynaptic boutons Whole-cell bleb recordings from layer 5 pyramidal neuron axons have shown that inactivation of low-threshold Kv1 subunitcontaining ion channel currents ("D-current") contribute, at least in
B

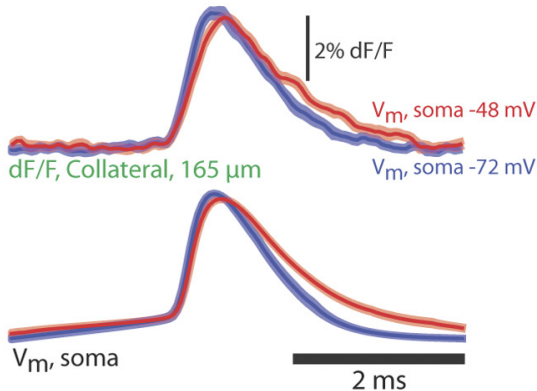

C

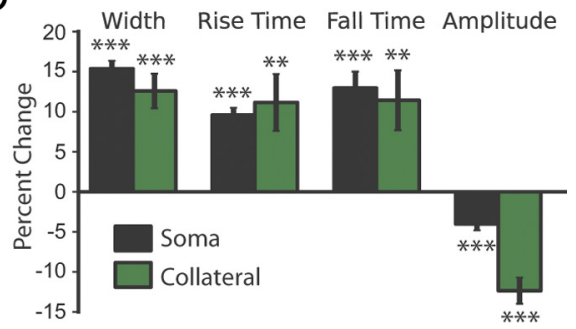

E

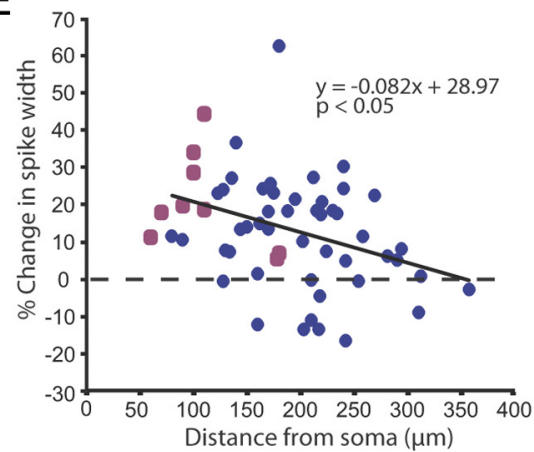

Figure 2. Steady-state somatic depolarization broadens action potentials in axon collaterals. $\boldsymbol{A}$, Confocal z-projection of a voltageFluorescence intensity traces (top) corresponding to spikes evoked with somatic current injection delivered after $>10$ s of steady-state (blue) and depolarization (red). Each trace is an average of 10 trials. Light blue and light red regions show the action potential in axon collaterals. Statistical comparisons shown are results of paired Student's ttests, ${ }^{* *} p<0.01,{ }^{* * *} p<0.001$. D, Spike width in main axon (pink) and collaterals (blue) following somatic depolarization plotted against the width of spikes preceded by somatic hyperpolarization. $\boldsymbol{E}$, Percentage change in spike width (FWHM) is negatively correlated with the total length of axon between the recording site and soma ( $n=50$ collaterals in 41 cells, $p=0.023$ ).

part, to broadening of axon potentials in the main axon with steadystate, subthreshold depolarization of the parent soma (Shu et al., 2007). To test whether D-current also contributes to the kinetics of action potential repolarization in axon collaterals and en passant presynaptic boutons, we examined the effects of low doses of $\alpha$-DTX (100 nM; $n=8$ cells) and 4-AP (40 $\mu \mathrm{M} ; n=8$ cells). Both $\alpha$-DTX and 4-AP caused dramatic broadening (250-300\% of control; 4-AP: mean $=255 \pm 25 \%$ SEM (standard error of the mean); $\alpha$-DTX: mean $=294 \pm 32 \%$ ) of action potentials in axon collaterals $(n=8)$ and putative presynaptic boutons ( $n=21$ boutons, Fig. $3 C, D$ ). As reported previously (Shu et al., 2007), the application of the Kvl channel antagonists had only a slight broadening effect on action potentials recorded in the soma (4-AP: mean $=113 \pm 4 \% ; \alpha$-DTX: mean $=107 \pm 2 \%$; Fig. $3 C$ ). Importantly, Kv1 channel blockade abolished the increase in axonal spike width with somatic subthreshold depolarization (Fig. $3 D ; n=8$ ), suggesting that under control conditions, subthreshold depolarization inactivates the D-current in axon collaterals, resulting in slowing of spike repolarization. In the soma, Kv1 channel blockade with $\alpha$-DTX did not abolish action potential broadening with subthreshold depolarization (Fig. 
A

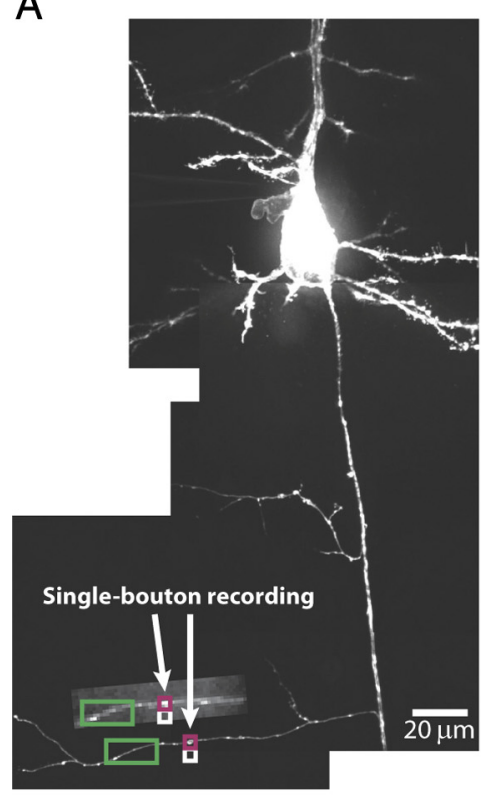

B
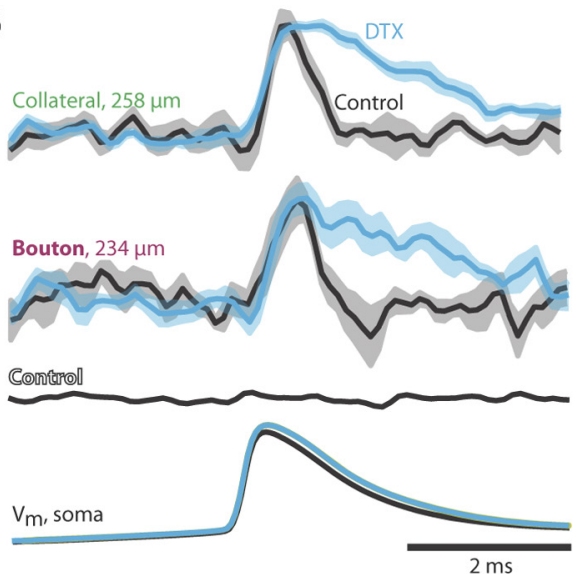

C

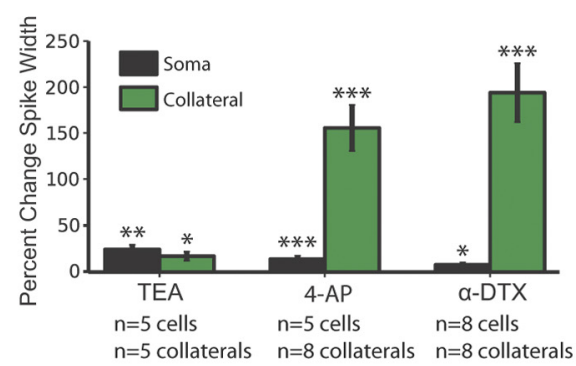

\section{E}

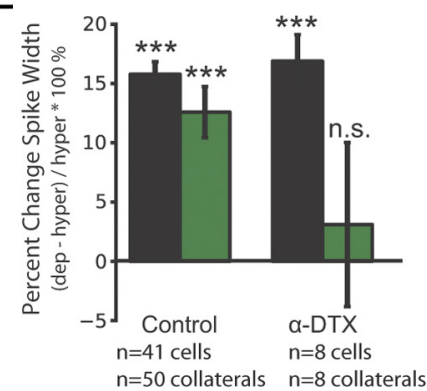

Figure 3. Spike repolarization depends on Kv1 currents in axon collaterals and presynaptic boutons. $A$, Confocal z-projection of a voltage-sensitive dye-filled layer 5 pyramidal cell. Inset shows the image of the axon collateral outlined in green taken with the CCD VSD camera. Bright puncta (i.e., magenta box) are putative en passant synaptic boutons. B, Bath application of $100 \mathrm{~nm} \alpha$-DTX (blue traces) causes significant slowing of the falling phase of spike related fluorescence transients in axon collaterals (top) and individual presynaptic boutons (middle, magenta box in $\boldsymbol{A}$ ), while the somatic spike (bottom) broadens only slightly. The optical signal from a pixel adjacent to the bouton shows the spatial specificity of the fluorescence signal from the presynaptic bouton (control, orange box in $\boldsymbol{A}$ ). Each trace shows an average of $7-8$ trials; light blue and gray regions indicate the SEM. C, Bar graph of the spike width (percentage change) for action potentials recorded in the soma (black) and collaterals (green) with bath application of $1 \mathrm{~mm}$ TEA, $40 \mu \mathrm{m}$ 4-AP, or $100 \mathrm{~nm} \alpha$-DTX. D, Bar graph of spike width recorded in the soma $(n=41)$, collaterals $(n=50)$, and individual presynaptic boutons ( $n=13$ ), follow steady-state somatic hyperpolarization (blue, green) or depolarization (red, purple) before and during bath application of $100 \mathrm{~nm} \alpha$-DTX. $\alpha$-DTX abolished depolarization-induced spike width broadening in axon collaterals. $\boldsymbol{E}$, Bar graph of the percentage change in spike width [(depolarized - hyperpolarized)/hyperpolarized $\times 100 \%$ ] for spikes recorded electrically with the somatic patch electrode (black) and optically in the collaterals (green). Spike broadening with subthreshold somatic depolarization is abolished with application of $100 \mathrm{~nm} \alpha$-DTX in the collaterals but not in the soma.

$3 D, E)$. Application of $1 \mathrm{~mm}$ tetraethylammonium, a concentration known to block $80 \%$ of $\mathrm{Kv} 3$ subunit-containing $\mathrm{K}^{+}$-channels (Rudy et al., 1999), caused only modest increases in both somatic (123 $\pm 5 \%$ of control) and axon collateral (116 $\pm 5 \%$ of control; $n=5)$ spike width (Fig. 3C), possibly through nonspecific blockade of non-Kv3 subunitcontaining channels.

\section{The role of collateral Kv1 channels in depolarization-induced spike broadening}

Assuming that action potential shape depends primarily on local voltage-dependent $\mathrm{Na}^{+}$and $\mathrm{K}^{+}$currents, the contribution of
D-current inactivation to action potential broadening at a given axonal location depends on two factors: the density of $\mathrm{Kvl}$ channels and the magnitude of subthreshold depolarization. However, it has also been suggested that local broadening of action potentials at the axon initial segment can propagate a significant distance down the axon (Kole et al., 2007). To assess whether the depolarization-induced spike broadening requires a significant contribution of D-current in the axon collaterals, we compared the effect of subthreshold depolarization on spike width in two model axons: Model 1 contains D-current in the AIS, nodes, and collateral, and Model 2 has D-current in the AIS only (Fig. 4).

The models each consist of a $500 \mu \mathrm{m}$ myelinated axon with a $20 \mu \mathrm{m}$ AIS and two nodes of Ranvier at 100 and $300 \mu \mathrm{m}$ with a $500 \mu \mathrm{m}$ collateral attached at the first node (Fig. 4A). Spikes were initiated in the AIS with brief ( $5 \mathrm{~ms}$ ) current pulses at the beginning and end of a long (20 s) subthreshold depolarization of the AIS through DC injection. In Model 1, which contains D-current in the AIS, nodes of Ranvier, and axon collateral, we found that long subthreshold depolarization of the AIS broadened the action potential in the AIS by $30 \%$ (Fig. $4 A, B$ ), and this effect decreased as the spike propagated down the main axon $(\lambda=152 \mu \mathrm{m})$. The broadening effect continued into the proximal collateral and decayed to baseline at 200 $\mu \mathrm{m}$ (Fig. $4 A, B$ ). Reducing $I_{\mathrm{Kd}}$ to $1 \%$ of the density used in Model 1 caused axon potentials to broaden to $144 \%$ of control (FWHM) in the AIS and 120-127\% in the axon collateral (Fig. 4A). While this qualitatively reproduced the experimental result of washing on DTX or 4-AP, it is significantly smaller than the 250-300\% of control increase seen in experiments. We assume that the reduced effect of D-current block on spike duration in the model with respect to our experimental results is due to the simplified nature of our model. We did not include calcium currents or persistent $\mathrm{Na}^{+}$currents, for example, which are known to contribute to the generation of prolonged action potentials. We chose to keep our model relatively simple, as opposed to putting speculative levels of ionic currents that are incompletely characterized, since our current model is sufficient to test the hypothesis that the presence of local D-current in the axon collateral is necessary to explain the expansion of action potential duration in axon collaterals with somatic depolarization. In Model 2, which contains D-current only in the AIS, subthreshold depolarization broadened the AIS spike width by $20 \%$, and this broadening propagated down the main axon with a length constant of $\lambda=136 \mu \mathrm{m}$. However, there was no broadening in the unmyelinated collateral, even in the compartment ad- 

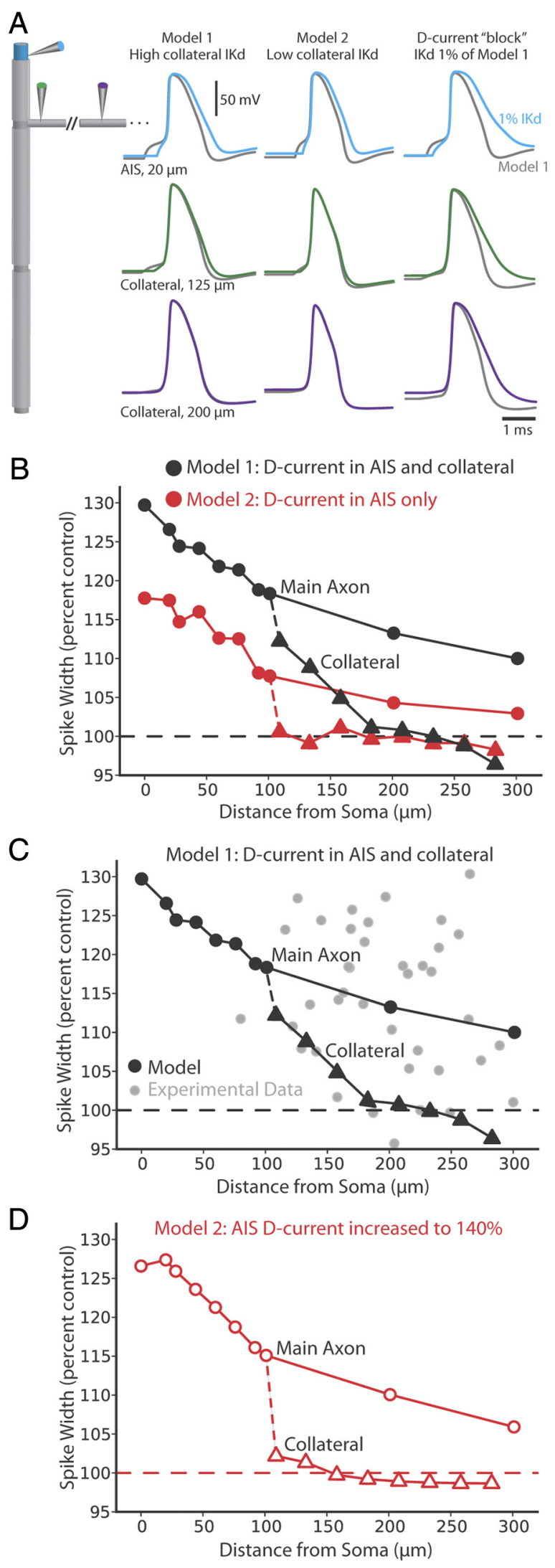

Figure 4. Depolarization-induced increases in collateral spike width requires local $D$-current. $A$, The axon model consists of a $500 \mu \mathrm{m}$ myelinated main axon with a $20 \mu \mathrm{m}$ initial segment and two nodes of Ranvier at 100 and $300 \mu \mathrm{m}$. A $500 \mu \mathrm{m}$ unmyelinated collateral issues from the first node of Ranvier, $80 \mu \mathrm{m}$ from the end of AIS. Two models are compared: (1) the AIS, nodes, and collateral contain $g_{\mathrm{Kd}}$ (left column), and (2) $g_{\mathrm{Kd}}$ is in the AIS only (middle jacent to the node from which it originates (Fig. 4A,B). Increasing the $\mathrm{D}$-current conductance in the AIS to $140 \%$ so that the increase of AIS spike width with somatic depolarization matched that of the AIS in Model 1 failed to increase collateral spike width with somatic depolarization (Fig. 4D). This result suggests that a high density of local D-current is necessary for the broadening of spike width in unmyelinated collaterals. Exploration of a wide range of values for the two $\mathrm{K}^{+}$currents responsible for spike repolarization $\left(I_{\mathrm{Kv}}\right.$ and $\left.I_{\mathrm{D}}\right)$ indicated that the ability of somatic depolarization to result in a lengthening of action potential duration in the axon collaterals requires a local dependence of action potential repolarization on $I_{\mathrm{D}}$ as well as the propagation of the steady shift in membrane potential into the region of collateral under consideration. Plotting the experimental data together with results from Model 1 with D-current in the collateral (Fig. $4 C$ ) shows that spike widths in most collaterals were broader than in the model, indicating that the model estimates a lower bound for $g_{\mathrm{Kd}}$ in the collateral.

\section{Discussion}

We demonstrate here that Kv1 subunit-containing channels are primarily responsible for repolarizing action potentials in the axon collaterals and their presynaptic en passant terminals of layer 5 cortical pyramidal neurons. Furthermore, we observed that subthreshold depolarization of the soma broadened action potentials in axon collaterals, an effect that was blocked with low concentrations of Kv1 channel antagonists. Using simple models with different distributions of D-current, we show that the broadening of action potentials in axon collaterals with subthreshold somatic depolarization requires the presence of $\mathrm{Kv} 1$ subunit-containing channels throughout the axon collaterals. Hence, broadening of spikes through the local inactivation of $\mathrm{Kvl}$ channels may play an important role in voltagedependent modulation of local synaptic transmission (Kole et al., 2007; Shu et al., 2007).

Far from simply disseminating all-or-none impulses from a presynaptic neuron to its targets, the cortical pyramidal neuron axonal arbor is a morphologically complex and specialized structure populated with ionic conductances diverse in both type and distribution that enable complex modulation of neuronal output (Debanne, 2004; Debanne et al., 2011). Indeed, many of the electrophysiological properties previously attributed to the soma, such as persistent $\mathrm{Na}^{+}$current, $\mathrm{M}$-current, $\mathrm{Ca}^{2+}$-activated $\mathrm{K}^{+}$ currents, and spike onset in fact arise primarily from the axon initial segment (Dodson and Forsythe, 2004; Astman et al., 2006; Inda et al., 2006; Lai and Jan, 2006; Yu et al., 2008, 2010). Simultaneous patch recording from the somata and axons of layer 5 pyramidal cells revealed that action potentials in the AIS repolar-

column). Spikes generated in the AIS (blue), proximal collateral (green), and distal collateral (purple) by brief ( $5 \mathrm{~ms}$ ) current injection at the AIS at the beginning (gray) and end (color) of a 20 s subthreshold DC injection at the AIS. The right column shows the effect of reducing $g_{\mathrm{Kd}}$ to $1 \%$ of the density used for Model 1 with no compensatory increase in $g_{\mathrm{kv}}$. Gray traces show APs generated with the original Model 1, and blue, green, and purple traces show the effect of D-current "block." B, Depolarization-induced change in spike width in the main axon (circles) and collateral (triangles) plotted as a function of distance from the AIS in Model 1 (black) with D-current in the collateral and Model 2 (red) without D-current in the collateral. C, Spike broadening for the main axon (circles) and collaterals (triangles) in Model 1 with D-current in the collateral plotted on top of experimental data (gray). D, Depolarization-induced changes in spike width for Model 2 (no D-current in the collateral) in which $g_{\mathrm{Kd}}$ was elevated in the AIS to increase spike broadening in the AIS to a degree similar to that of the high $g_{\mathrm{Kd}}$ model. Even with D-current elevated in the AIS, depolarization-induced spike broadening fails to propagate into the collateral if the D-current is localized to only the AIS. 
ize largely through activation of Kv1.2 $\alpha$-subunit-containing $\mathrm{K}^{+}$ channels, a current contributing relatively little to repolarization of action potentials in the soma (Kole et al., 2007; Shu et al., 2007; Yu et al., 2008, 2010). However, it is not yet possible to use electrodes to directly determine which conductances mediate spike repolarization in axon collaterals.

Utilizing recent significant advances in the signal-to-noise ratio of single-cell voltage-sensitive dye imaging, for the first time we directly measured the shape of action potentials propagating in axon collaterals and presynaptic terminals of neocortical pyramidal cells. We observed that action potentials propagating through the collaterals, like in the main axon (Kole et al., 2007), rise and repolarize faster than in the axon initial segment and soma. Thus the characteristics of action potential waveforms invading presynaptic terminals can deviate significantly from the spike recorded at the soma. In addition, application of Kv1, but not Kv3, channel antagonists dramatically slowed action potential repolarization in the collaterals and presynaptic boutons, similar to previous findings in the main axon of layer 5 pyramidal cells (Kole et al., 2007; Shu et al., 2007). Interestingly, the block of Kv1-subunit-containing ion channels broadens the action potential in the soma only slightly even in the presence of a greatly broadened action potential in the main axon and collaterals (e.g., Fig. $3 B$ ). These results reinforce the notion that, at least for portions of the neuron where action potentials are actively generated (as opposed to passively propagated), the properties of action potential repolarization rely almost entirely upon the local $\mathrm{K}^{+}$ currents available, and not those at more distal locations. One study reported that broadening action potentials at the AIS with the local application of 4-AP or $\alpha$-DTX can result in the propagation of broadened action potentials down the main axon (Kole et al., 2007). However, for this broadening of the action potential at the AIS to affect synaptic transmission, it would have to propagate into the unmyelinated axon collaterals, where presynaptic terminals are located. In our model axon, we observed that AIS depolarization-induced broadening of action potentials in the local collaterals depended upon the presence of the D-current in the axon collaterals. This result, combined with our observation that collateral spike widths are highly sensitive to block of Kv1containing channels, suggests that it is the local dependence of spike repolarization on Kv1 channels in the axon collateral and presynaptic boutons that mediate depolarization-induced spike width facilitation. This mechanism of facilitation of synaptic transmission, therefore, is completely dependent on the propagation of depolarization of the membrane potential between action potentials (e.g., resting $V_{\mathrm{m}}$ ) from the soma into the presynaptic terminals of axon collaterals. Computational models of cortical pyramidal cell axon arbors suggest that prolonged (e.g., seconds) somatic depolarization can propagate with a length constant of $\sim 300-350 \mu \mathrm{m}$ into the axonal arbor, potentially influencing between 150 and 1500 nearby synapses of layer 5 pyramidal cells (Shu et al., 2006).

The ability of D-current to modulate action potential duration with changes in subthreshold membrane potential may significantly affect local synaptic transmission. Specifically, a small (5-15 mV) subthreshold somatic depolarization can significantly enhance the average amplitude of EPSPs evoked by single spikes in both postsynaptic pyramidal (Shu et al., 2006) and GABAergic (Zhu et al., 2011) neurons, resulting in membrane-potential modulation of local network dynamics. What mechanisms underlie EPSP enhancement by presynaptic membrane potential? Broadening of action potentials in presynaptic terminals is likely the main mechanism since small increases in presynaptic spike duration can strongly enhance EPSC amplitude (Geiger and Jonas, 2000). Indeed, a significant part of the depolarization-induced enhancement of synaptic transmission occurs with a time constant similar to the slow kinetics of D-current inactivation (Shu et al., 2007; Zhu et al., 2011). Our data confirm that action potentials propagating in collaterals bearing en passant presynaptic terminals broaden with steady-state somatic depolarization at distances of up to $300 \mu \mathrm{m}$ from the soma. Broadening of action potentials with the bath application of $\alpha$-DTX or 4-AP, blockers of the D-current, can result in strong enhancement of pyramidalpyramidal and pyramidal-interneuron synaptic communication. This enhancement may preclude the synaptic enhancement observed with depolarization of cortical pyramidal cells, suggesting that depolarization-induced spike broadening in the axon may mediate the depolarization-induced enhancement of synaptic transmission (Kole et al., 2007; Zhu et al., 2011).

In addition to axonal spike broadening, we also found that somatic depolarization decreased the amplitude of action potentials, presumably due to voltage-gated sodium channel inactivation. While amplitude reduction may work against synaptic facilitation by decreasing voltage-dependent activation of $\mathrm{Ca}^{2+}$ channels, this may be countered by the increase in driving force for $\mathrm{Ca}^{2+}$ entry owing to the reduced action potential peak amplitude. Lengthening the falling phase of action potential generation has a disproportionately large effect on $\mathrm{Ca}^{2+}$ entry into the presynaptic terminal, and subsequent transmitter release, since it is during this period of the spike that the $\mathrm{Ca}^{2+}$ currents are most active (Sabatini and Regehr, 1997; Qian and Saggau, 1999; Geiger and Jonas, 2000). Monitoring baseline and spike-triggered presynaptic $\mathrm{Ca}^{2+}$ levels in presynaptic terminals has revealed that depolarization of the soma can result in increases in both of these for nearby $(<200 \mu \mathrm{m})$ terminals (Yu et al., 2010). Although the increases in spike-triggered $\mathrm{Ca}^{2+}$ levels may be explained by the changes in axonal spike shape with somatic depolarization, as observed here, increases in baseline $\mathrm{Ca}^{2+}$ levels presumably result from low-level activation of $\mathrm{P} / \mathrm{Q}$ or $\mathrm{N} \mathrm{Ca}^{2+}$ channels by subthreshold depolarization (Awatramani et al., 2005; Bender et al., 2010; Yu et al., 2010; Christie et al., 2011). Together, our data indicate that broadening and amplitude reduction of action potentials may contribute to increases in spike-triggered calcium influx in presynaptic terminals, and in combination with increased baseline calcium, increase the probability of neurotransmitter release (Bender et al., 2010; Yu et al., 2010; Christie et al., 2011)

Our results demonstrate that Kv1 channels contribute significantly to spike repolarization throughout the local axonal arbor of layer 5 pyramidal neurons, including collaterals participating in local synaptic transmission. Furthermore, blockade of depolarization-induced spike broadening with $\alpha$-DTX indicates that inactivation of axonal D-current contributes significantly to spike broadening and potentially increases transmitter release probability. This mechanism may be widely used in the nervous system since Kv1 subunits dominate axon terminals in many cell types (Debanne et al., 2011), where they prevent aberrant action potential generation (Dodson et al., 2003). Mossy fiber boutons, for instance, contain a high density of Kv1.4 subunit-containing channels that strongly influence spike duration and transmitter release (Geiger and Jonas, 2000). In contrast, layer 5 pyramidal neuron axon collaterals and presynaptic boutons were relatively insensitive to Kv3 channel blockade with TEA, a current identified in the axon terminals of many other cells types, both excitatory and inhibitory (Debanne et al., 2011).

There is likely a diversity of ion channels contributing to axonal action potential generation in the wide variety of cortical neuronal subtypes, since cortical cell types differ markedly in the properties of action potential generation at their somata (Shu et al., 2007; Kole et al., 2008; Yu et al., 2008). Which ionic channels 
are involved in action potential repolarization and membrane potential excitability in axons, collaterals, and presynaptic boutons of the different cell types will significantly influence how the flow of synaptic activity associated with behavior influences synaptic transmission in local cortical networks. Only through the understanding of each neuron as a whole, and how each part of the neuron interacts with the network, will we begin to understand neocortical function and dysfunction.

\section{References}

Agmon A, Connors BW (1991) Thalamocortical responses of mouse somatosensory (barrel) cortex in vitro. Neuroscience 41:365-379.

Ahern GP, Hsu SF, Klyachko VA, Jackson MB (2000) Induction of persistent sodium current by exogenous and endogenous nitric oxide. J Biol Chem 275:28810-28815.

Alle H, Geiger JR (2006) Combined analog and action potential coding in hippocampal mossy fibers. Science 311:1290-1293.

Antic S, Cohen LB, Lam YW, Wachowiak M, Zecevic D, Zochowski M (1999) Fast multisite optical measurement of membrane potential: three examples. FASEB 13 [Suppl 2]:S271-S276.

Astman N, Gutnick MJ, Fleidervish IA (2006) Persistent sodium current in layer 5 neocortical neurons is primarily generated in the proximal axon. J Neurosci 26:3465-3473.

Awatramani GB, Price GD, Trussell LO (2005) Modulation of transmitter release by presynaptic resting potential and background calcium levels. Neuron 48:109-121.

Bender KJ, Ford CP, Trussell LO (2010) Dopaminergic modulation of axon initial segment calcium channels regulates action potential initiation. Neuron 68:500-511.

Canepari M, Djurisic M, Zecevic D (2007) Dendritic signals from rat hippocampal CA1 pyramidal neurons during coincident pre- and postsynaptic activity: a combined voltage- and calcium-imaging study. J Physiol 580:463-484.

Canepari M, Vogt K, Zecevic D (2008) Combining voltage and calcium imaging from neuronal dendrites. Cell Mol Neurobiol 28:1079-1093.

Canepari M, Willadt S, Zecevic D, Vogt KE (2010) Imaging inhibitory synaptic potentials using voltage sensitive dyes. Biophys J 98:2032-2040.

Christie JM, Chiu DN, Jahr CE (2011) Ca(2+)-dependent enhancement of release by subthreshold somatic depolarization. Nat Neurosci 14:62-68.

Colbert CM, Pan E (2002) Ion channel properties underlying axonal action potential initiation in pyramidal neurons. Nat Neurosci 5:533-538.

Cooper EC, Milroy A, Jan YN, Jan LY, Lowenstein DH (1998) Presynaptic localization of Kv1.4-containing A-type potassium channels near excitatory synapses in the hippocampus. J Neurosci 18:965-974.

Dallas ML, Atkinson L, Milligan CJ, Morris NP, Lewis DI, Deuchars SA, Deuchars J (2005) Localization and function of the Kv3.1b subunit in the rat medulla oblongata: focus on the nucleus tractus solitarii. J Physiol 562:655-672.

Debanne D (2004) Information processing in the axon. Nat Rev Neurosci 5:304-316.

Debanne D, Campanac E, Bialowas A, Carlier E, Alcaraz G (2011) Axon physiology. Physiol Rev 91:555-602.

Djurisic M, Antic S, Chen WR, Zecevic D (2004) Voltage imaging from dendrites of mitral cells: EPSP attenuation and spike trigger zones. J Neurosci 24:6703-6714.

Dodson PD, Forsythe ID (2004) Presynaptic K+ channels: electrifying regulators of synaptic terminal excitability. Trends Neurosci 27:210-217.

Dodson PD, Billups B, Rusznák Z, Szûcs G, Barker MC, Forsythe ID (2003) Presynaptic rat Kv1.2 channels suppress synaptic terminal hyperexcitability following action potential invasion. J Physiol 550:27-33.

Engel D, Jonas P (2005) Presynaptic action potential amplification by voltage-gated $\mathrm{Na}(+)$ channels in hippocampal mossy fiber boutons. Neuron 45:405-417.

Foust A, Popovic M, Zecevic D, McCormick DA (2010) Action potentials initiate in the axon initial segment and propagate through axon collaterals reliably in cerebellar Purkinje neurons. J Neurosci 30:6891-6902.

Geiger JR, Jonas P (2000) Dynamic control of presynaptic Ca2+ inflow by fast-inactivating $\mathrm{K}+$ channels in hippocampal mossy fiber boutons. Neuron 28:927-939.

Hasenstaub A, Otte S, Callaway E, Sejnowski TJ (2010) Metabolic cost as a unifying principle governing neuronal biophysics. Proc Natl Acad Sci U S A 107:12329-12334.
Hines ML, Carnevale NT (1997) The NEURON simulation environment. Neural Comput 9:1179-1209.

Holthoff K, Zecevic D, Konnerth A (2010) Rapid time course of action potentials in spines and remote dendrites of mouse visual cortex neurons. J Physiol 588:1085-1096.

Hu W, Tian C, Li T, Yang M, Hou H, Shu Y (2009) Distinct contributions of $\mathrm{Na}(\mathrm{v}) 1.6$ and $\mathrm{Na}(\mathrm{v}) 1.2$ in action potential initiation and backpropagation. Nat Neurosci 12:996-1002.

Huang H, Trussell LO (2008) Control of presynaptic function by a persistent $\mathrm{Na}(+)$ current. Neuron 60:975-979.

Inda MC, DeFelipe J, Muñoz A (2006) Voltage-gated ion channels in the axon initial segment of human cortical pyramidal cells and their relationship with chandelier cells. Proc Natl Acad Sci U S A 103:2920-2925.

Kole MH, Letzkus JJ, Stuart GJ (2007) Axon initial segment Kvl channels control axonal action potential waveform and synaptic efficacy. Neuron 55:633-647.

Kole MH, Ilschner SU, Kampa BM, Williams SR, Ruben PC, Stuart GJ (2008) Action potential generation requires a high sodium channel density in the axon initial segment. Nat Neurosci 11:178-186.

Lai HC, Jan LY (2006) The distribution and targeting of neuronal voltagegated ion channels. Nat Rev Neurosci 7:548-562.

Lambe E, Aghajanian GK (2001) The role of Kv1.2-containing potassium channels in serotonin-induced glutamate release from thalamocortical terminals in rat frontal cortex. J Neurosci 21:9955-9963.

Leão RM, Kushmerick C, Pinaud R, Renden R, Li GL, Taschenberger H, Spirou G, Levinson SR, von Gersdorff H (2005) Presynaptic Na ${ }^{+}$channels: locus, development, and recovery from inactivation at a high-fidelity synapse. J Neurosci 25:3724-3738.

Mainen ZF, Joerges J, Huguenard JR, Sejnowski TJ (1995) A model of spike initiation in neocortical pyramidal neurons. Neuron 15:1427-1439.

McCormick DA (1992) Neurotransmitter actions in the thalamus and cerebral cortex and their role in neuromodulation of thalamocortical activity. Prog Neurobiol 39:337-388.

Monaghan MM, Trimmer JS, Rhodes KJ (2001) Experimental localization of Kv1 family voltage-gated $\mathrm{K}^{+}$channel $\alpha$ and $\beta$ subunits in rat hippocampal formation. J Neurosci 21:5973-5983.

Qian J, Saggau P (1999) Modulation of transmitter release by action potential duration at the hippocampal CA3-CA1 synapse. J Neurophysiol 81:288-298.

Rudy B, Chow A, Lau D, Amarillo Y, Ozaita A, Saganich M, Moreno H, Nadal MS, Hernandez-Pineda R, Hernandez-Cruz A, Erisir A, Leonard C, VegaSaenz de Miera E (1999) Contributions of Kv3 channels to neuronal excitability. Ann N Y Acad Sci 868:304-343.

Sabatini BL, Regehr WG (1997) Control of neurotransmitter release by presynaptic waveform at the granule cell to Purkinje cell synapse. J Neurosci 17:3425-3435.

Shu Y, Hasenstaub A, Duque A, Yu Y, McCormick DA (2006) Modulation of intracortical synaptic potentials by presynaptic somatic membrane potential. Nature 441:761-765.

Shu Y, Yu Y, Yang J, McCormick DA (2007) Selective control of cortical axonal spikes by a slowly inactivating $\mathrm{K}+$ current. Proc Natl Acad Sci U S A 104:11453-11458.

Southan AP, Robertson B (2000) Electrophysiological characterization of voltage-gated $\mathrm{K}^{+}$currents in cerebellar basket and Purkinje cells: Kvl and Kv3 channel subfamilies are present in basket cell nerve terminals. J Neurosci 20:114-122.

Wang H, Kunkel DD, Schwartzkroin PA, Tempel BL (1994) Localization of Kv1.1 and Kv1.2, two K channel proteins, to synaptic terminals, somata, and dendrites in the mouse brain. J Neurosci 14:4588-4599.

Weiser M, Bueno E, Sekirnjak C, Martone ME, Baker H, Hillman D, Chen S, Thornhill W, Ellisman M, Rudy B (1995) The potassium channel subunit $\mathrm{KV} 3.1 \mathrm{~b}$ is localized to somatic and axonal membranes of specific populations of CNS neurons. J Neurosci 15:4298-4314.

Yu Y, Shu Y, McCormick DA (2008) Cortical action potential backpropagation explains spike threshold variability and rapid-onset kinetics. J Neurosci 28:7260-7272.

Yu Y, Maureira C, Liu X, McCormick D (2010) P/Q and N channels control baseline and spike-triggered calcium levels in neocortical axons and synaptic boutons. J Neurosci 30:11858-11869.

Zhu J, Jiang M, Yang M, Hou H, Shu Y (2011) Membrane potentialdependent modulation of recurrent inhibition in rat neocortex. PLoS Biol 9:e1001032. 